\title{
Can your dog read your mind? Understanding the causes of canine perspective taking
}

\author{
Monique A. R. Udell • Nicole R. Dorey • \\ Clive D. L. Wynne
}

Published online: 4 June 2011

(C) Psychonomic Society, Inc. 2011

\begin{abstract}
Prior studies have documented the domestic dog's (Canis lupus familiaris) sensitivity to human attentional state, including a tendency to preferentially beg for food from attentive individuals and an ability to selectively perform forbidden behaviors when humans are not looking. Due to the success of dogs on perspective-taking tasks, some have hypothesized that domestic dogs may have theory of mind, or the ability to infer what other individuals know. Here we provide the first evidence that nondomesticated canids, grey wolves (Canis lupus), are also sensitive to human attentional state under some conditions. We also demonstrate that dogs do not display an undifferentiated sensitivity to all visual cues of attentional state. Rather, dogs are more sensitive to stimuli encountered in their home environment. Some dogs perform poorly on perspective-taking tasks. These findings have important implications for the interpretation of research designed to understand complex social cognition across species.
\end{abstract}

Keywords Wolves · Dogs · Canis lupus familiaris · Canis lupus · Perspective taking - Social cognition - Theory of mind $\cdot$ Domestication $\cdot$ Dog shelter $\cdot$ Learning

Recent studies have identified a remarkable range of human-like social behaviors in the domestic dog (Canis

M. A. R. Udell $(\bowtie) \cdot$ N. R. Dorey • C. D. L. Wynne Department of Psychology, University of Florida, P.O. Box 112250, Gainesville, FL 32611, USA

e-mail: moniqueudell@gmail.com

C. D. L. Wynne

Kyung Hee University,

Suwon, South Korea lupus familiaris). Pet dogs have been shown capable of responding to human body language, verbal commands, and attentional states to a degree that has equaled, or in some cases surpassed, our closest primate relatives, chimpanzees (for reviews, see Hare \& Tomasello, 2005; Miklósi, Topál, \& Csányi 2007; Udell \& Wynne, 2008). Furthermore, pet dogs have performed at high levels on five of the seven theory-of-mind methodologies discussed by Heyes (1998), including imitation (Miller, Rayburn-Reeves, \& Zentall 2009; Range, Virányi, \& Huber 2007; Topál, Byrne, Miklósi, \& Csányi 2006a), deception (Petter, Musolino, Roberts, \& Cole 2009), role taking (Miklósi, Polgárdi, Topál, \& Csányi 2000; Topál, Erdõhegyi, Mányik, \& Miklósi 2006b), seeing and knowing or guesser-knower (Cooper et al., 2003), and seeing and attending or begging studies (Cooper et al., 2003; Gácsi, Miklósi, Varga, Topál, \& Csányi 2004). Even more astounding are the repeated successful demonstrations of theory-of-mind abilities in dogs, while evidence of theoryof-mind-like skills in nonhuman primates has been much more elusive (see Heyes, 1998; Povinelli \& Eddy, 1996; Povinelli \& Vonk, 2003).

Success on theory-of-mind tasks has traditionally led to interpretations that suggest that "an animal with a theory of mind believes that mental states play a causal role in generating behavior and infers the presence of mental states in others by observing their appearance and behavior under various circumstances" (Heyes, 1998, p. 102). However, many conventional theory-of-mind methodologies, and the complex interpretations of their results, have faced criticism because it is debatable whether they are capable of empirically demonstrating awareness of another's knowledge state (Heyes, 1998; Penn \& Povinelli, 2007). This has been especially true when the species in question are nonverbal. Instead, subjects may simply be responding to 
environmental cues, the presence or absence of certain stimuli, or even the behavioral cues of the other individual. Therefore, some have argued that a theory-of-mind account is not necessary to predict and explain these sometimes complex behaviors in experimental or natural settings (e.g., Baum, 1998). Others have argued that such tests simply fail to capture evidence of theory of mind, asserting that associative accounts do not rule out the possibility that a subject might still be sensitive to the attentional state of others (e.g., Gómez, 1998). It has also been argued that some species might possess some components of theory of mind while lacking others (Call \& Tomasello, 2008), or that modifications made to traditional tasks could provide more definitive results (Penn \& Povinelli, 2007). While the majority of research in this area has been conducted with primates, and thus criticism has often been directed at studies suggesting the presence of theory-of-mind in monkeys or apes, it should be noted that the criticisms are equally valid when considering theory-of-mind claims across species.

In the face of the current debates, perspective-taking tasks have been considered among the most promising for the study of theory-of-mind and related forms of social cognition (Heyes, 1998). The goal of these tasks is to determine whether a subject is able to behave in accordance with another individual's access to relevant information.

The begging task is a perspective-taking methodology in which a subject has to select one of two similar humans to approach for food. An individual is given a choice between an attentive person and a person rendered unable to see the animal in some way. In this scenario, subjects must respond to cues of human attentional state to identify the person willing to produce food-in this case, the attentive experimenter.

At present, two studies have tested domestic dogs' sensitivity to human attentional state in a begging task. Interestingly, the method used to obscure the inattentive human's vision has seemed to reliably correspond with the relative success of dog subjects on the task. Gácsi et al. (2004) reported that pet dogs were more likely to beg for food from an attentive woman than from an inattentive woman; however, performance was more accurate when the inattentive woman turned her back than when she wore a blindfold. Likewise, Cooper et al. (2003) found that dogs were more likely to ignore a person whose face was obscured behind a book than a bucket. The fact that a dog's performance may vary based on the visible stimulus associated with human inattention raises interesting questions about the origin of perspective-taking behavior in pet domestic dogs. Do domestic dogs succeed on perspectivetaking tasks because they possess a theory of mind, or can their successes be explained as the outcome of associative processes, such as conditioning, within their natural environment?
In some cases, it has been argued that domestic dogs evolved "human-like social skills" as a by-product of domestication (Hare, Brown, Williamson, \& Tomasello 2002; Hare \& Tomasello, 2005). This has included the assertion that "dogs do not acquire these skills mainly because of their experiences in ontogeny" (Riedel, Schumann, Kaminski, Call, \& Tomasello 2008, p. 1007) and that "human exposure has no major effect on dogs' ability to use humangiven communicative cues and that this skill therefore represents a special adaptation in dogs which is present from early age" (Riedel et al., 2008, p. 1012). If it is true that "the unusual skills of domestic dogs in using human communicative cues most likely evolved during and as a result of human domestication" (Hare et al., 2010, p. E6), then all healthy populations of domestic dogs should be expected to outperform nondomesticated canids on human attentionalstate tasks. Furthermore, this should hold true independent of the age, condition, home environment, or experience level of the dog under test (Hare et al., 2002; Miklósi et al., 2003; Riedel et al., 2008).

While a hypothesis suggesting that domestication may predispose dogs to attend to human stimuli and a hypothesis predicting the importance of ontonogenic or environmental influences are not mutually exclusive, in practice the proximate variables that contribute to the human-oriented social behavior of domestic dogs have often been neglected or overlooked (see Udell, Dorey, \& Wynne 2010b, for a review). It is also possible that a genetic predisposition acquired through domestication is not necessary to explain the behavior of dogs. Instead, both domesticated and undomesticated canids may learn to beg preferentially from attentive humans on the basis of prior experiences in which these individuals were more likely to offer treats, attention, or other reinforcers. If this is the case, pet dogs should be more sensitive to cues of attentional state common to their home environments. For example, one might predict that pet dogs would treat familiar stimuli such as a human reading a book, magazine, or other document as a salient predictor of inattention, while demonstrating less responsiveness to unfamiliar cues, such as a person with a bucket over her head. Shelter dogs, despite belonging to the same (sub)species as pet dogs, would be expected to behave in accordance with their relevant life experiences, which include less recent experience with people terminating attention by reading in their presence, for example. Likewise, socialized wolves would be expected to perform above chance under conditions in which they have had experience with relevant human stimuli, and less so in conditions in which they have not. However, if wolves were successful under any condition, this would demonstrate that neither domestication nor the presence of a specially evolved human-like social cognition is necessary to explain sensitivity to human attentional state. 
The purpose of the present study is to test whether the rearing and living environment of the canid subject (shelter or human home) or species identity (dog or wolf) has the greater impact on an individual's performance. If perspective-taking behavior is learned on the basis of experiences with relevant stimuli in an individual's current living environment, performance would be expected to vary according to the correspondence of an individual's experiences and the condition under test. Additional experience on a condition would be expected to improve the performance of individuals on that condition, assuming that they are socialized to humans and motivated to accomplish the task. Such findings would be consistent with the predictions of the two-stage hypothesis, which emphasize the important interactions between phylogeny and ontogeny in multiple areas of canine social cognition (Udell et al., 2010b).

These predictions cannot directly rule out the possibility that successful canid subjects might also be able to infer the mental states of others. If the above predictions are correct, however, it would suggest that (1) experience and environment are important in the development of perspective-taking behavior and (2) the mechanisms responsible for perspective taking are not exclusive to domesticated canids, nor would domestication be sufficient to predict canid behavior on perspective-taking tasks. On the other hand, if perspective-taking behavior were to be identified in different populations of dogs, independent of environment, experience, and occluder type, but not shared by human-socialized wolves under matched conditions, one might argue that domestication resulted in a special adaptation, such as a predisposition to look at human faces (Miklósi et al., 2003) or more human-like social skills (Hare \& Tomasello, 2005), not present in their undomesticated counterparts. In the present study, we compare the performance of pet domestic dogs, shelter dogs, and wolves on a begging task in order to test these predictions.

\section{Experiment 1: Perspective taking in a begging task}

In Experiment 1, we tested the perspective-taking abilities of four groups of canids: tame hand-raised wolves, pet domestic dogs tested outdoors like the wolves, pet dogs tested indoors, and dogs tested indoors at the local animal shelter where they resided. Subjects had to choose between an attentive human ("seer") and a human whose visual attention was blocked ("blind") in one of four ways: the human had her back turned; she held a book over her face; she had a bucket over her head; or she held a camera over her eyes.
Method

Subjects A group of 8 tame, hand-raised adult wolves residing at Wolf Park, Battle Ground, Indiana, were tested individually in an outdoor arena. All wolves were reared and socialized according to the specifications of Klinghammer and Goodman (1987). The experimenters were inside the enclosure with the wolf during testing. Of the wolves, 6 (Miska, Marion, Ayla, Tristan, Ruedi, and Renki) had previously served in a study testing their ability to follow human pointing gestures (Udell, Dorey, \& Wynne 2008a). All wolves had been hand raised by staff from 10-14 days of age and had interacted with humans daily, receiving food treats directly from humans on a regular basis. A group of pet domestic $\operatorname{dogs}(N=18)$ of varying breeds were tested in a situation as similar as possible to that in which the wolves had been tested (outdoors, physical isolation, but with distant auditory and olfactory contact to conspecifics in other enclosed areas on the property). Another group of pet $\operatorname{dogs}(N=$ 17) was tested indoors, isolated from conspecifics and the other distractions inherent to an outdoor testing environment. Domestic dogs from a county dog shelter $(N=17)$ were tested indoors. Only dogs recorded as strays, as opposed to rescues or pet surrenders, were utilized. Although the exact life history of strays prior to their arrival at the shelter could not be determined, the designation "stray" signifies that the subject had not been surrendered to the shelter by an owner or by another animal facility. Instead, these individuals had been found away from a home environment, typically in an open field or on the roadside. A requirement for all subjects was that they readily approach and take food from the experimenter; this eliminated the possibility that a subject might be too afraid or distracted to participate in the study. This behavior is also characteristic of individuals with prior socialization to humans; minimally, some exposure to humans during the sensitive period for social development, which ends around 14 weeks of age (Scott \& Fuller, 1965). Therefore, none of the dogs in this study, including the stray dogs living in the shelter, were considered feral.

To limit possible generalization effects, dogs were tested on no more than two experimental conditions each, except for 8 dogs that did not complete the second condition due to satiation or the owner's schedule. In these cases, an additional subject was tested on the remaining condition (Table 1). All wolves participated in all four conditions because the number of wolves available was limited. A break of between an hour and a day separated the first two and the second two testing conditions for the wolves. Generalization across conditions did not appear to occur, based on the results of the study. 
Table 1 Name, age, sex, breed, and condition assignment information for all subjects

\begin{tabular}{|c|c|c|c|c|c|c|c|c|c|}
\hline Name & Age (years) & Sex & Breed & Group & Testing Location & Condition 1 & Condition 2 & Condition 3 & Condition 4 \\
\hline Rockie & 0.7 & $\mathrm{M}$ & Golden-doodle & Pet dog & Indoors & Back turned & Book & & \\
\hline Bell & 0.5 & $\mathrm{~F}$ & Corgi & Pet dog & Indoors & Back turned & Book & & \\
\hline R. Noodle & 2.0 & M & Chihuahua & Pet dog & Indoors & Back turned & Book & & \\
\hline Molly & 4.0 & $\mathrm{~F}$ & Mix & Pet dog & Indoors & Back turned & Book & & \\
\hline Andy & 2.5 & M & Rat terrier & Pet dog & Indoors & Bucket & Back turned & & \\
\hline Toby & 3.0 & M & Chihuahua & Pet dog & Indoors & Bucket & Back turned & & \\
\hline Samara & 1.0 & $\mathrm{~F}$ & Vizsla & Pet dog & Indoors & Bucket & Back turned & & \\
\hline Leo & 1.0 & M & Pomeranian & Pet dog & Indoors & Camera & Bucket & & \\
\hline Taz & 4.0 & $\mathrm{~F}$ & Jack Russell & Pet dog & Indoors & Book & Camera & & \\
\hline John Luke & 0.8 & M & Border Collie & Pet dog & Indoors & Book & Camera & & \\
\hline Mateo & 3.0 & M & Basset Hound & Pet dog & Indoors & Camera & Bucket & & \\
\hline Comet & 4.0 & M & Golden Retriever & Pet dog & Indoors & Book & Camera & & \\
\hline Roxie & 2.5 & $\mathrm{~F}$ & Beagle mix & Pet dog & Indoors & Book & Lost motivation & & \\
\hline Otis & 3.0 & M & Terrier & Pet dog & Indoors & Bucket & Back turned & & \\
\hline Hollywood & 3.0 & M & Min. Pinscher & Pet dog & Indoors & Camera & Lost motivation & & \\
\hline Scooby Doo & 9.0 & M & Ital. Greyhound & Pet dog & Indoors & Camera & Bucket & & \\
\hline Zoe & 5.0 & $\mathrm{~F}$ & Cattle Dog & Pet dog & Indoors & Camera & Bucket & & \\
\hline Honey & 2.5 & $\mathrm{~F}$ & Cocker Spaniel & Pet dog & Outdoors & Back turned & Book & & \\
\hline Saddie & 0.6 & $\mathrm{~F}$ & Lab. Retriever mix & Pet dog & Outdoors & Back turned & Book & & \\
\hline Bambam & 2.0 & M & Golden-doodle & Pet dog & Outdoors & Back turned & Book & & \\
\hline Bugsy & 2.0 & M & Puggle & Pet dog & Outdoors & Book & Camera & & \\
\hline Wiley & 4.0 & M & Chihuahua mix & Pet dog & Outdoors & Book & Camera & & \\
\hline Jeaka & 3.5 & $\mathrm{~F}$ & Australian Shepherd & Pet dog & Outdoors & Bucket & Back turned & & \\
\hline Bingo & 11.0 & M & Mix & Pet dog & Outdoors & Back turned & Book & & \\
\hline Luna & 3.0 & $\mathrm{~F}$ & Sheltie & Pet dog & Outdoors & Book & Camera & & \\
\hline Jacks & 1.5 & M & Lab. Retriever & Pet dog & Outdoors & Book & Camera & & \\
\hline Pretzel & 1.5 & $\mathrm{~F}$ & Boston Terrier & Pet dog & Outdoors & Bucket & Back turned & & \\
\hline Cliff & 5.0 & M & Brittany Spaniel & Pet dog & Outdoors & Bucket & Lost motivation & & \\
\hline Tucker & 2.0 & M & Terrier & Pet dog & Outdoors & Bucket & Back turned & & \\
\hline Honey 2 & 0.7 & $\mathrm{~F}$ & Corgi & Pet dog & Outdoors & Bucket & Back turned & & \\
\hline Loki & 0.6 & $\mathrm{~F}$ & Lab. Retriever & Pet dog & Outdoors & Camera & Lost motivation & & \\
\hline Remus & 2.5 & M & Daschund & Pet dog & Outdoors & Camera & Lost motivation & & \\
\hline Dolly & 5.0 & $\mathrm{~F}$ & Mix & Pet dog & Outdoors & Bucket & - & & \\
\hline Mavrick & 4.0 & M & Min. Pinscher & Pet dog & Outdoors & Camera & Bucket & & \\
\hline Gabby & 7.0 & $\mathrm{~F}$ & Schnauzer & Pet dog & Outdoors & Camera & Bucket & & \\
\hline 33 & 1.5 & M & Lab. Retriever mix & Shelter dog & Indoors & Back turned & Book & & \\
\hline 35 & 0.5 & M & Pit Bull & Shelter dog & Indoors & Back turned & Book & & \\
\hline 36 & - & $\mathrm{M}$ & Lab. Retriever mix & Shelter dog & Indoors & Back turned & Book & & \\
\hline 40 & 0.6 & M & Pit Bull mix & Shelter dog & Indoors & Back turned & Book & & \\
\hline 27 & 0.4 & M & Pit Bull mix & Shelter dog & Indoors & Bucket & Back turned & & \\
\hline 28 & 3 & $\mathrm{M}$ & Pit Bull mix & Shelter dog & Indoors & Bucket & Back turned & & \\
\hline 30 & 2 & $\mathrm{~F}$ & Lab. Retriever mix & Shelter dog & Indoors & Bucket & Back turned & & \\
\hline 32 & 1 & $\mathrm{~F}$ & Pit Bull & Shelter dog & Indoors & Bucket & Back turned & & \\
\hline $32 \mathrm{~B}$ & 2 & M & Bulldog & Shelter dog & Indoors & Book & Lost motivation & & \\
\hline 39 & 0.8 & $\mathrm{~F}$ & Lab. Retriever mix & Shelter dog & Indoors & Book & Camera & & \\
\hline $30 \mathrm{~B}$ & 1 & $\mathrm{~F}$ & Lab. Retriever mix & Shelter dog & Indoors & Book & Camera & & \\
\hline 31 & 1 & $\mathrm{~F}$ & Chow mix & Shelter dog & Indoors & Book & Camera & & \\
\hline 41 & 0.8 & M & Lab. Retriever mix & Shelter dog & Indoors & Camera & Bucket & & \\
\hline 55 & 1.5 & M & Rottweiler & Shelter dog & Indoors & Camera & Bucket & & \\
\hline $35 \mathrm{~B}$ & 1 & $\mathrm{~F}$ & Pit Bull mix & Shelter dog & Indoors & Camera & Bucket & & \\
\hline 37 & 1.5 & M & German shepherd & Shelter dog & Indoors & Camera & Lost motivation & & \\
\hline $40 \mathrm{~B}$ & 0.5 & M & German shepherd & Shelter dog & Indoors & Camera & Bucket & & \\
\hline Ayla & 4 & $\mathrm{~F}$ & N/A & Wolf & Outdoors & Back turned & Book & Camera & Bucket \\
\hline
\end{tabular}


Table 1 (continued)

\begin{tabular}{|c|c|c|c|c|c|c|c|c|c|}
\hline Name & Age (years) & Sex & Breed & Group & Testing Location & Condition 1 & Condition2 & Condition 3 & Condition 4 \\
\hline Chetan & 13 & M & $\mathrm{N} / \mathrm{A}$ & Wolf & Outdoors & Back turned & Book & Camera & Bucket \\
\hline Marion & 10 & $\mathrm{~F}$ & N/A & Wolf & Outdoors & Back turned & Book & Camera & Bucket \\
\hline Miska & 12 & $\mathrm{M}$ & N/A & Wolf & Outdoors & Back turned & Book & Camera & Bucket \\
\hline Renki & 4 & M & N/A & Wolf & Outdoors & Back turned & Book & Camera & Bucket \\
\hline Ruedi & 4 & $\mathrm{M}$ & N/A & Wolf & Outdoors & Back turned & Book & Camera & Bucket \\
\hline Tristan & 10 & $\mathrm{M}$ & $\mathrm{N} / \mathrm{A}$ & Wolf & Outdoors & Back turned & Book & Camera & Bucket \\
\hline Wolfgang & 3 & M & N/A & Wolf & Outdoors & Back turned & Book & Camera & Bucket \\
\hline
\end{tabular}

"Lost motivation" indicates that the subject could not be further tested after failing a test of food motivation.

Pretraining All subjects were given up to eight opportunities to approach the experimenters and eat food from their hands four times before testing. During this phase, the experimenters stood close together, face to face, with the relevant occluder in the hand farthest from the subject (Fig. 1a). When the experimenters were in place, on the assistant's count of three, the experimenters simultaneously called the subject's name to obtain its attention. If the subject approached the experimenters upon release, both individuals simultaneously presented a piece of food for consumption. Food rewards included 2$\mathrm{cm}$ cubes of Spam, Beggin Strips, and Pet Botanics dog food rolls. Two dogs from the pound did not meet this pretest criterion within eight trials and were not included in the study.

Testing conditions For each experimental trial, one experimenter was designated the inattentive or blind individual, and the other was designated the attentive individual, the seer. The identity of the experimenters as seer or blind was predetermined pseudorandomly for each trial, such that for each subject in each condition both experimenters served as the seer or the blind experimenter equally often. Both the seer and the blind experimenter were instructed to remain still in a forward-facing orientation, except during the backturned condition, throughout each trial. The seer was additionally instructed to visually track the subject throughout the trial so that her eyes were visible and eye contact could be made if initiated by the subject. Once experimental testing began, if the subject approached the seer directly upon release, the seer would present her food to the subject for consumption. The blind experimenter never provided food, although both individuals always held food in their free hand on every trial.

There were four testing conditions in this experiment, each corresponding to a specific stimulus indicating attentional state. In the back-turned condition, the target experimenter oriented toward the subject with her back against the fence, while the blind experimenter faced the fence with her back to the subject. In all other conditions, both experimenters oriented toward the subject holding the condition-designated occluder; however, only the Blind experimenter placed this occluder over her eyes or head, blocking her view of the subject. In the book condition, the seer held an open book (approximately $23 \times 30 \mathrm{~cm}$ ) up to her chest with one hand and looked at the subject while the blind experimenter held a book in front of her face. In the bucket condition, the seer held an opaque off-white plastic wastepaper container (approximately $34 \times 23 \times 20 \mathrm{~cm}$ ) on
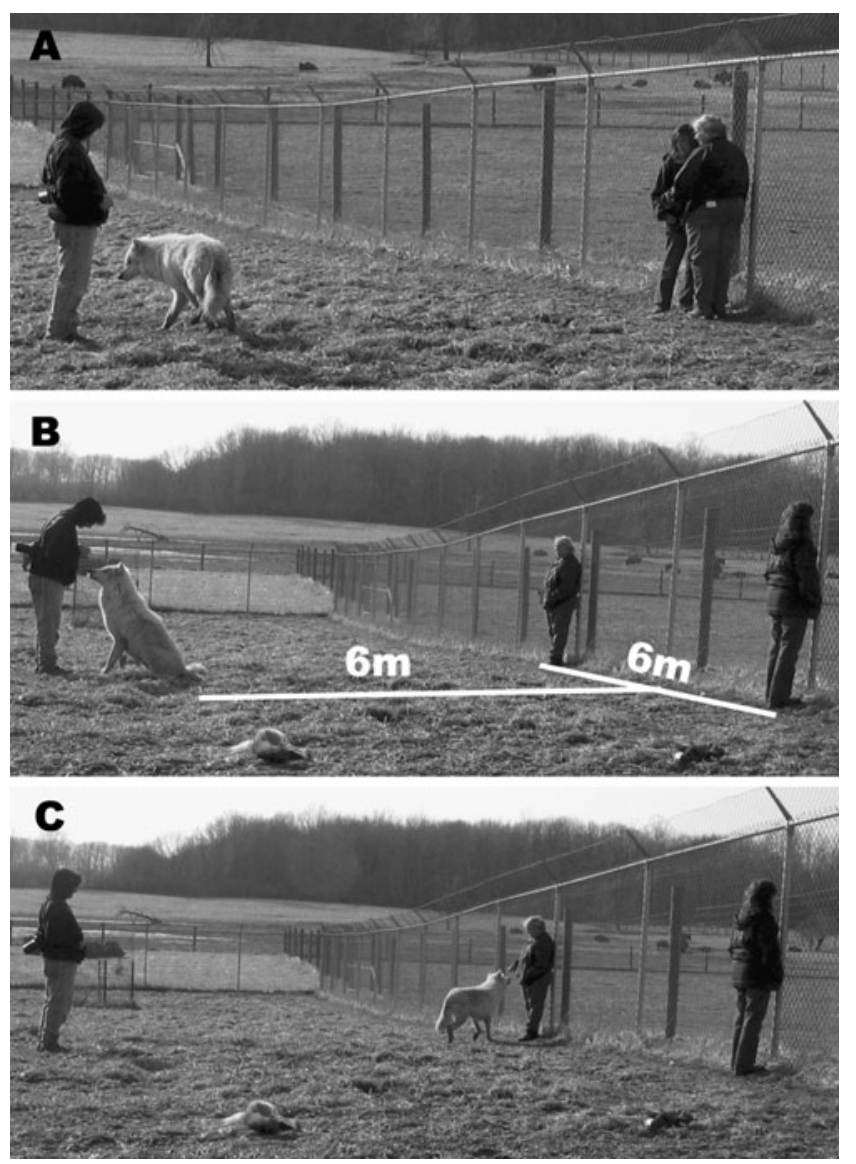

Fig. 1 Testing layout and procedure. (a) Pretraining trials. (b) Experimental trials, with layout measurements. (c) Rewarding a correct choice. The condition pictured is back turned 
her shoulder next to her head, and the blind experimenter placed an identical bucket over her head and held it in place so that the bottom rim came to just above her mouth. This allowed the majority of her head to be covered while leaving the quality of the experimenter's voice unaffected when calling for the subject's attention. In the camera condition, the seer held a camera (Canon Powershot, approximately $7 \times 10 \mathrm{~cm}$ ) over her mouth, and the blind experimenter held the camera in front of her eyes. Each subject received 10 testing trials for each condition it participated in (see Table 1). The two experimenters alternated left-right locations between trials.

Testing procedure The experimenters stood $6 \mathrm{~m}$ apart against a fence (outdoors) or wall (indoors). Both were provisioned in their pockets with the same food used in pretraining. The experimental trials began immediately after pretraining. The assistant remained equidistant from both experimenters and restrained the subject $6 \mathrm{~m}$ back from the midpoint between them (see Fig. 1b).

Before each trial, the assistant informed the experimenters of their role as the seer or the blind individual. Once the experimenters were in position with their condition-specific occluder in place, the assistant counted to three, at which time both experimenters simultaneously called the subject's name (or a term such as "puppy," for subjects without known names). They repeated this up to five times at approximately 30 -s intervals as long as the subject remained more than $2 \mathrm{~m}$ back from either experimenter. The timing and occurrence of experimenter behavior was determined by the assistant, who initiated each additional simultaneous call by counting to three.

A choice was recorded when the subject touched either experimenter or remained within $1 \mathrm{~m}$ of either experimenter for $3 \mathrm{~s}$. Once this criterion was met, the assistant did one of two things. If the seer was chosen, the trial was scored as correct and the assistant said "feed," marking the choice and instructing the experimenter to immediately present her food to the subject for consumption (Fig. 1c). If the blind experimenter was chosen, the trial was scored as incorrect and the subject was not given any food; the assistant immediately called the subject back to the starting position and began the next trial. If the subject failed to approach either experimenter within $3 \mathrm{~min}$, this was recorded as "no choice" and the trial was terminated. No-choice trials were also scored as incorrect responses. If three incorrect responses were made in succession, a pretraining trial was carried out to test for motivation. No dog failed a test of motivation within its first testing condition. Dogs that demonstrated decreased motivation in the pretraining trials given before the start of their second condition were not allowed to continue (see Table 1).
Statistical analysis Nonparametric statistics were utilized throughout due to our conservative sample sizes. A Kruskal-Wallis test was used to determine whether there were significant differences between the performance of the canid groups for each condition. We then utilized a onesample signed rank sum test to determine whether each canid group performed significantly better than chance on each of the four occluder conditions. In addition, the individual performance of each subject on each condition was compared to chance using a binomial test. A score of 8 or more correct trials out of 10 was considered successful performance on the condition $(p \leq .05)$. A two-tailed alpha level of .05 was adopted for all statistical tests.

\section{Results}

Performance varied significantly between canid groups in the back-turned condition (Kruskal-Wallis: $H_{3}=11.55, p=$ $.009)$, with pet dogs tested outdoors and wolves performing most accurately (median trials correct, 9/10) and shelter dogs performing least accurately (median trials correct, $7 /$ $10)$ at the group level. However, all canid groups still performed significantly above chance on the back-turned condition (one-sample signed rank sum test: each group, $n=8, T=0, p \leq .03)$. Average performance also varied between canid groups in the book condition (KruskalWallis: $H_{3}=7.84, p=.05$ ). In this condition, pet dogs tested indoors (one-sample signed rank sum test: $n=8$, $T=0, p=.008$ ) and pet dogs tested outdoors (one-sample signed rank sum test: $n=8, T=1.50, p=.02$ ) were the only groups to perform significantly above chance. Pet dogs tested indoors also performed above chance in the bucket condition (one-sample signed rank sum test: $n=8$, $T=2.50, p=.02$ ), but statistically significant differences in performance across groups were not detected in either the bucket (Kruskal-Wallis: $H_{3}=2.60, p=.46$ ) or camera (Kruskal-Wallis: $H_{3}=12.14, p=.54$ ) conditions. Figure 2 shows the group median and individual performance for subjects in each canid group across all four experimental conditions.

\section{Discussion}

The hypothesis that domestication is sufficient for dogs to engage in perspective taking is rendered problematic by the observation that dogs' performance on these tests depends on the type of occluder used and on the life conditions of the canid. Furthermore, the success of wolves on one condition demonstrates that domestication is not essential for above-chance performance under all conditions.

The alternative position is that domestic dogs and other socialized canids are not taking the mental perspective of the seeing or blind individual, but instead making a 
Fig. 2 Begging task performance across groups and conditions for Experiment 1. Each black dot represents an individual subject's performance (the number of correct choices out of 10 experimental trials). The bars represent each group's median score. An asterisk (*) indicates that group performance was significantly better than chance (one-sample signed rank sum test: $p<.05)$ The trend lines indicate $50 \%$ chance
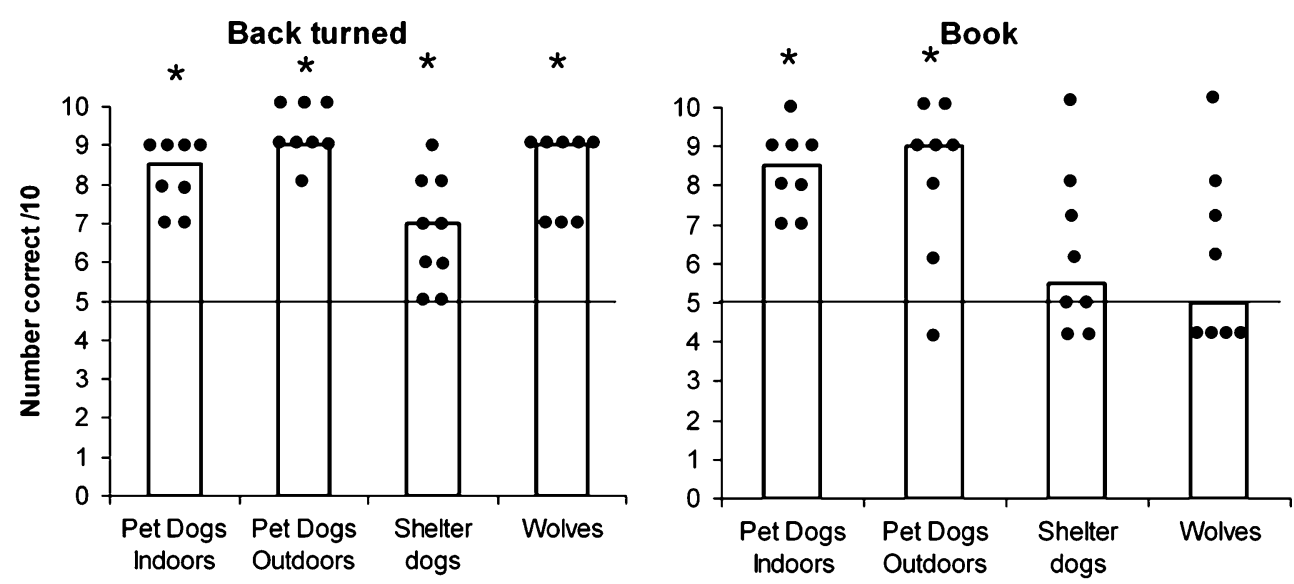

Camera

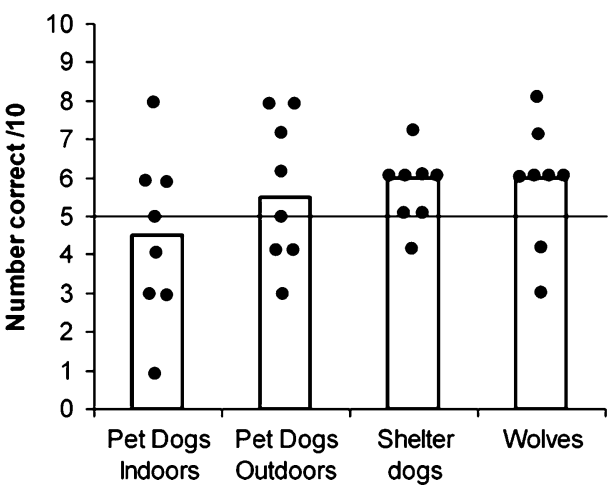

\section{Bucket}

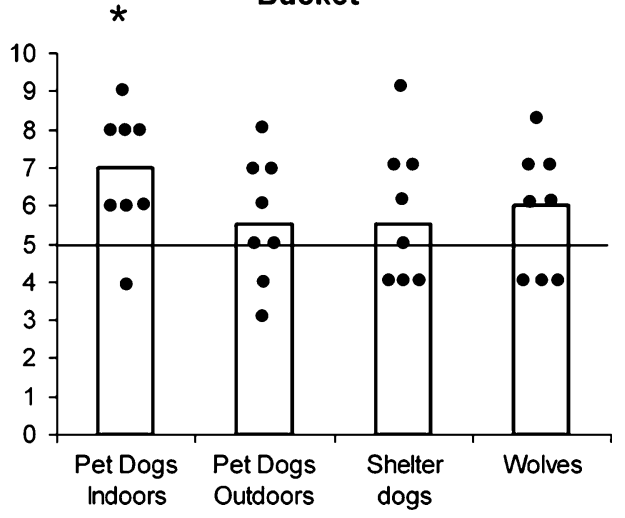

discriminative choice based on past reinforcement histories, in which certain human actions or orientations served as predictors of reinforcement upon approach. This hypothesis is consistent with the patterns of success and failure found in this study. A human turning her back and terminating delivery of food treats is a cue with which individuals in all groups have likely had prior experience, and the majority of subjects across all groups met criterion in this condition. Furthermore, both groups of pet dogs performed above chance on the begging task with a book as the occluder, a finding consistent with Cooper et al. (2003), whereas shelter dogs and wolves were typically not successful on this condition. This is consistent with the likely recent experiences of the different groups of dogs and wolves.

The camera condition was originally chosen because it was a relatively small occluder that both wolves and dogs in all groups had some experience with. However a subject's failure to discriminate between a person holding a camera over her eyes and an individual with unoccluded eyes is difficult to interpret. It is possible that the size of the occluder made the camera a less salient stimulus, leading to a failure to discriminate between the two experimenters. On the other hand, humans can and do see through the lens of a camera and may even actively reinforce dogs or wolves for orienting toward them if they are the subject of the photograph. Therefore, the failure to discriminate between experimenters in this condition might even be considered the correct response. More research is needed to interpret the responses of canids toward objects that could serve as occluders but functionally are not.

Most striking was the finding that only 7 out of 32 subjects reliably begged from the seer when the alternative experimenter had a bucket over her head. If dogs possessed a generalized sensitivity to attentional state, this condition should have provided a definitive contrast between the seeing and blind experimenters. However relevant this distinction might seem to humans, it is likely that most dogs and wolves have not had the necessary life experiences to teach them that a bucket over a human's head signals reduced attention. Dogs may rarely witness their owners with a bucket on their head; none of the owners volunteering dogs in our study reported such displays.

Interestingly, in the original nonhuman primate begging study carried out by Povinelli and Eddy (1996), chimpanzees also failed to preferentially beg from an attentive individual when the inattentive individual had his vision occluded by a bucket (this was also true when the occluder was a blindfold or screen). And, as for all canid groups in the present study, an experimenter turning his back to the chimpanzee subject was the most reliable way to signal the absence of reinforcement or inattention (a result that has now been replicated in several species of nonhuman 
primate: Kaminski, Call, \& Tomasello 2004; Povinelli \& Eddy, 1996). However, the chimpanzee data also provide a noteworthy contrast to the behavior of the pet dogs. While the chimpanzees failed to spontaneously make a discrimination when any inanimate occluder blocked the experimenter's vision, pet dogs have been shown to reliably beg from an attentive person when the other individual has her face covered by a book (Cooper et al., 2003; Exp. 1 of the present study). This suggests that the opportunity for exposure to a specific occluder type, even if informally in the home environment, can serve as an important predictor of performance in a begging task. For chimpanzees lacking this prior exposure, additional experimental training was found to improve performance on the begging task, even when the stimulus was an inanimate occluder such as a bucket (Povinelli \& Eddy, 1996).

If inadequate experience explains the poor performance of canids on a condition for which they have little relevant prior exposure, then one would expect subjects to show improvement over the course of additional trials. In Experiment 2, we exposed dogs and wolves to additional trials in the bucket condition to see whether a difference in the behavior of the canids in the presence of the seer versus the blind experimenter might be more pronounced in a learning paradigm.

\section{Experiment 2: Flexibility in perspective-taking tasks}

Experiment 2 had two goals: (1) to see whether canid performance in the bucket condition would improve with additional exposure to the contingencies, and (2) to determine whether the two choice options, seer and blind experimenter, were truly functionally equivalent stimuli prior to the additional exposure. In other words, would canids learn the reinforcement contingencies at the same rate, independent of whether the reinforced response was approaching the attentive or inattentive individual?

To address these questions, we tested 12 naive dogs and retested 8 human-socialized wolves on a modified bucket condition of the begging task in which each subject was exposed to twice as many trials as in Experiment 1. Because wolves and pet dogs tested outdoors had previously performed equivalently in the bucket condition of the begging task (group mean scores: dogs outdoors, 5.63/10; wolves, 5.75/10; 1 successful individual in each group), in Experiment 2 they were combined into a single canid group.

Half of the subjects - 6 dogs and 4 wolves-were put into the "seer" condition, where the seeing experimenter reinforced begging with food. The remaining 6 dogs and 4 wolves were placed in the "blind" condition, where begging from the person with the bucket on her head was reinforced with food. Comparing the rates of acquisition of responsiveness to blind and seeing people permitted a more sensitive test of any difference in the canids' responsiveness in these two conditions.

Past research has shown that pet dogs can learn to perform above chance on object choice tasks requiring the use of novel gestures within 10 experimental trials (Udell, Giglio, \& Wynne 2008b) and to look at a human for food reinforcement in as few as 3 trials (Bentosela, Barrera, Jakovcevic, Elgier, \& Mustaca 2008). Wolves have also been shown to learn to respond appropriately to human cues with additional exposure (Virányi et al., 2008) in cases in which they were initially unsuccessful. Here, we investigated whether dogs and wolves tested outdoors would show the same rapid flexibility for learning about cues predicting a human's willingness to provide food in response to begging.

\section{Method}

Subjects Two groups of 12 pet dogs living in human homes and 8 tame, hand-raised adult wolves residing at Wolf Park, Battle Ground, Indiana, were tested outdoors under the same conditions as Experiment 1 (Table 2). All 12 pet dogs were naive to the experimental task and had not participated in Experiment 1. One dog from the seer condition and 1 from the blind condition only completed 10 and 8 trials, respectively, before failing a test of motivation. The performance of these 2 dogs could not be used to assess the effect of additional experimental trials, and they are excluded from the statistical analysis. The 10 remaining dogs, 5 from the seer group and 5 from the blind group, all completed testing.

The 8 wolves tested in Experiment 2 had previously participated in Experiment 1. While it was not possible to

Table 2 Name, breed, age, sex, and group designations of pet domestic dogs in Experiment 2

\begin{tabular}{lllll}
\hline Group & Name & Breed & Age & Sex \\
\hline Seer & Petey & Poodle & 10 & $\mathrm{M}$ \\
Seer & Van & Shar-Pei Mix & 1 & $\mathrm{M}$ \\
Seer & Lulubelle & Dachshunds & 7 & $\mathrm{~F}$ \\
Seer & Mousse & Labrador Retriever & 2 & $\mathrm{M}$ \\
Seer & Mollie & Weimaraner & 5 & $\mathrm{~F}$ \\
Seer & Sadie & Labrador Retriever & 4 & $\mathrm{~F}$ \\
Blind & Sonny & Goldendoodle & 4 & $\mathrm{~F}$ \\
Blind & Truet & Australian Shepherd & 0.6 & $\mathrm{M}$ \\
Blind & Jake & Labrador Retriever & 1 & $\mathrm{M}$ \\
Blind & Moon & Labrador Retriever & 1 & $\mathrm{M}$ \\
Blind & Colt & Unknown & 8 & $\mathrm{M}$ \\
Blind & Lily & Labrador Retriever & 3 & $\mathrm{~F}$ \\
\hline
\end{tabular}


obtain naive human-socialized wolves for the second experiment, 7 of the wolf subjects were tested 9 months after the completion of Experiment 1, and the 8th wolf, Wolfgang, was tested 12 months after the end of the first experiment. This extended delay was implemented to reduce the effect of the 10 previous trials of exposure to the task from the year before. In addition, the four lowestperforming wolves from Experiment 1-Ayla, Chetan, Marion, and Ruedi-were placed in the seer group, where begging from the seeing experimenter was reinforced. The 4 highest-performing wolves on the bucket condition from Experiment 1-Tristan, Miska, Renki, and Wolfgangwere placed in the blind group, where begging from the blind individual was now reinforced.

Materials and procedure The pretraining, testing, and choice designations were identical to those described for the bucket condition of Experiment 1. The only exceptions were that up to 20 trials were given to each subject and that the correct response was determined by the group to which each subject was assigned: seer or blind. Criterion was set at 8 correct responses out of the previous 10 trials; the subject finished the experiment once it met criterion or had received 20 experimental trials, whichever came first.

As in Experiment 1, the assistant informed the experimenters of their role as the seer or the blind individual before each trial. Once the experimenters were in position with their buckets in place- the seer with the bucket on her outside shoulder and the blind experimenter with the bucket over her head - the assistant counted to three, at which time both experimenters simultaneously called the subject's name. They repeated this up to five times at approximately 30 -s intervals as long as the subject remained more than $2 \mathrm{~m}$ back from either experimenter. The timing and occurrence of experimenter behavior was determined by the assistant, who initiated each additional simultaneous call by counting to three out loud.

A choice was recorded when the subject touched either experimenter or remained within $1 \mathrm{~m}$ of either experimenter for $3 \mathrm{~s}$. Once this criterion was met, the assistant did one of two things. If the condition-specific target individual was chosen, the trial was scored as correct and the assistant said "feed," marking the choice and instructing the experimenter to immediately present her food to the subject for consumption (Fig. 1c). In the seer condition, the correct choice was approaching the seer, and the blind individual never provided food. In the blind condition, the correct choice was approaching the blind experimenter, and the seer never provided food. Therefore, if the conditionspecific incorrect experimenter was chosen, the trial was scored as incorrect and the subject was not given any food; the assistant immediately called the subject back to the starting position and began the next trial. Although the subject received food only if the correct choice was made, both the seer and blind individual held food in their free hand on every trial, to ensure that the presence or absence of food was not being used as an additional cue. Because the assistant verbally directed all experimenter behavior, independent of condition, it did not matter whether the experimenter was able to see or not during a trial. Neither the blind nor the seeing experimenter fed the subject or removed her bucket prior to instruction from the assistant. If the subject failed to approach either experimenter within $3 \mathrm{~min}$, this was recorded as "no choice" and the trial was terminated. No-choice trials were scored as incorrect responses. If three incorrect responses were made in succession, a pretraining trial was carried out to test for motivation.

Statistical analysis A binomial test was used to determine whether a subject performed significantly above chance in the first 10 trials of testing. The numbers of individuals from the two conditions who met the success criterion8 out of the previous 10 trials correct by the end of the study - were compared using Fisher's exact test. A twotailed alpha level of .05 was adopted for both statistical tests.

\section{Results and discussion}

In total, 3 out of the 6 dogs and 1 of the 4 wolves (4/10 canids) in the seer group performed significantly above chance on the begging task within the first 10 trials (binomial tests: $p \leq .05$ ). In contrast, only 1 of the dogs and none of the wolves (1/10 canids) from the blind group initially performed above chance on the task.

Of the 9 canids in each group, a total of 6 from the seer group and only 1 from the blind group met criterion within 20 trials. The difference between the numbers of successes in the groups was statistically significant (Fishers exact test: $p=.05)$. The trend in performance was observable both for canids as a whole and for dogs and wolves considered separately (Fig. 3).

Experiment 2 showed that both wolves and dogs are capable of rapid improvement of their detection of the individual giving treats when that person has an unobscured view of the canid; however, when the blind individual was reinforcing the animals, only 1 canid improved significantly within 20 trials of exposure.

\section{General discussion}

These findings demonstrate for the first time that wolves, in addition to domestic dogs, are capable of succeeding in a perspective-taking task. Both wolves and dogs held and 


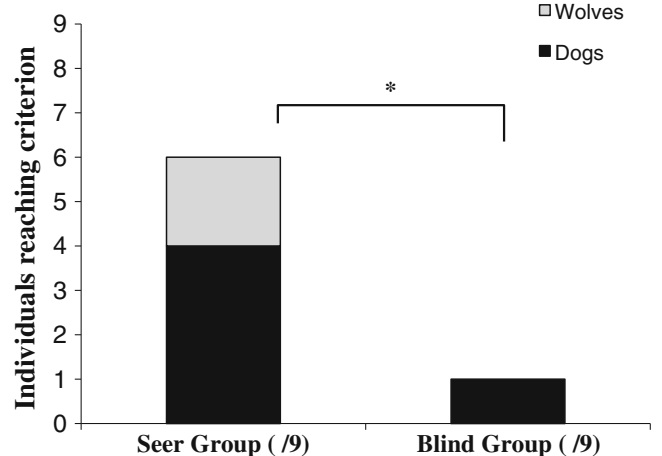

Fig. 3 Numbers of individual subjects meeting criterion with additional trials. The bars indicate the numbers of successful individuals out of a total of 9 canids - 5 dogs and 4 wolves - from each group. To reach criterion, a subject had to make the correct response in 8 out of the previous 10 trials. Subjects were given 20 trials to meet criterion. The asterisk $(*)$ indicates that the numbers of subjects meeting criterion in the seer and blind groups were significantly different (Fisher's exact test: $p=.05$ )

tested under different conditions were capable of responding to at least one type of attentional cue (back turned), demonstrating that the capacity to behave in accordance with a human's attentional state is shared by both domesticated and nondomesticated canids. In addition, both wolves and pet domestic dogs are able to rapidly improve their performance on perspective-taking conditions in which they are initially unsuccessful.

Experiment 1 further demonstrated that some populations of dogs - for example, dogs living in a shelter-are not initially as responsive to some stimuli predicting human attentional state as are those dogs living as pets. This is not to say that dogs living in a shelter could not learn to make the appropriate response, given additional training; in fact, it has been demonstrated that dogs living in a shelter can learn to utilize even a challenging human point to locate a target rapidly if given additional exposure to the task (Udell, Dorey, \& Wynne 2010a). What our results do suggest is that even for healthy, human-socialized dogs, life experiences common to strays or a shelter environment predict a different initial level of responsiveness to stimuli that predict a human's attentional state or willingness to provide reinforcement than is found in pet dogs.

It should be noted that a number of factors aside from lifetime exposure to specific stimuli or occluders might influence the performance of dogs living in a shelter environment, including stress or the possibility of an underlying wariness of the situation or handler. Although more research is needed to better understand the full range of variables that could result in performance differences, identifying that such differences exist is in itself an important outcome.

It is impossible to fully know or have control over the life history of animals before their arrival in a shelter. While this does limit the range of questions that can be asked about this population, it should be acknowledged that the same consideration is true of many important target populations, including pets not owned by researchers, human subjects, and wild animals. Outside of a laboratory setting, one can never be sure that the full accurate history of a subject is accounted for. While this fact can provide extra challenges to the design and interpretation of research, it does not negate the importance of understanding how individuals of a species behave in naturally occurring environments, nor does it justify the failure to ask the questions that can and should be addressed.

In the case of the present study, the most conservative assumption - that shelter dogs are just former pets with life experiences identical to those of the pet population prior to living in a shelter-would possibly lead to the most important and startling outcome: that only a short duration of time in a less enriched environment, with reduced exposure to humans and human actions, produces a significant reduction in the likelihood that a dog will succeed in a perspective-taking task. However, future research is necessary to directly manipulate and evaluate the lifetime factors that are most predictive of the behavioral differences that we have now identified in this naturally occurring population.

It was also true that several individual pet dogs and wolves were able to rapidly learn to beg from the seer, but not from the blind experimenter, in the bucket condition presented in Experiment 2. One possible explanation for this learning bias is that canids have an inherited predisposition for responding to attentive individuals, independent of experience. Since the direction of this trend was the same for wolves and for domestic dogs, both might be said to share this predisposition. This is contrary to previous suggestions that dogs, but not wolves, have been prepared to look at human faces through the process of domestication (Miklósi et al., 2003). However, such a predisposition toward attentive individuals would not explain why different groups of canids differed in success based on the types of occluder utilized in Experiment 1 or in previous experiments of this type (Cooper et al., 2003; Gácsi et al., 2004). If all canids possessed generalized perspectivetaking skills, it would be expected that both dogs and wolves should have preferred the seer in all conditions, regardless of which occluder the blind experimenter was holding.

Different levels of success when different occluders are employed instead implicate environment and experience as important variables contributing to canid success on these tasks. For pet dogs, this means that a book may serve as a useful signal for human inattention, while a bucket over a human's head, though overt, may hold little meaning until the dog has had a chance to test the contingencies 
associated with its presence. This explanation is further supported by the evidence that $80 \%$ of pet dogs and $50 \%$ of wolves tested learned to preferentially beg from the seer within just 20 trials of exposure in Experiment 2.

It might be argued that the distinction between the seeing and blind experimenters was more obvious in some conditions than in others. This hypothesis gains credibility from the finding that the size of a human gesture correlates with the performance of dogs on object choice tasks (Udell et al., 2008b) and leads to the prediction that all canid groups should perform best on the back-turned condition, followed by the bucket condition, then the book, and finally the camera. All groups did perform above chance on the back-turned condition, while none performed above chance on the camera condition. Size ordering, however, fails to explain the pattern of performance observed in the bucket and book conditions. Both groups of pet dogs performed more accurately in the book condition than in the bucket condition, while the other groups of canids (shelter dogs and wolves) failed on both. Thus, neither the size of the cue, the perspective of the human, nor the status of a canid as domesticated or not accurately predicts the performance of subjects on this task in all cases, challenging the predictions of the domestication hypothesis and of a generalized theory-of-mind explanation.

A second alternative is that canids are flexible in their choice strategy and learn about conditions under which begging does or does not pay off. It is natural to assume that the canids in this task are being asked to make one choice: "From which of these two people should I beg?" In Experiment 2, if the canids were exclusively learning about the proportional payoff of the seer versus the blind experimenter in the experimental setting, then subjects in each group should have learned to preferentially beg from the reinforced experimenter at about the same rate. Instead, both dogs and wolves in the seer group rapidly learned to approach the attentive individual providing food, but those in the blind group did not learn to preferentially approach the reinforced person in the same number of trials.

An alternative possibility is that each option was treated as an independent choice - that is, "Should I beg from the individual with an unblocked face?" and "Should I beg from the individual with the occluded face?" If this were the case, one would expect that a dog's lifetime learning history would guide behavior in the presence of each new set of stimuli predicting a human's willingness or unwillingness to provide food. Thus, in Experiment 2, approaching the human with the unblocked face might have been learned more rapidly because attentive humans have provided reinforcement in the past. Exclusive preference would not be blocked by a comparable history with inattentive individuals. Conversely, learning to approach an individual with an obscured face might occur more slowly (1) because the subject has less reinforced begging experiences in the presence of individuals whose faces are obscured by buckets and (2) because the act of begging from the other option, the seer, might take longer to extinguish, since a competing history of reinforcement for begging from attentive individuals in the home environment likely exists. Therefore, in the blind condition, responding may remain split between the two choice options for a greater number of trials.

In other words, it might not be the case that begging from a seeing individual is inherently more intuitive; instead, subjects might have difficulty reversing a previously reinforced response even after the contingencies of reinforcement have changed. In fact, resistance to reversal learning has been demonstrated in a number of species across experimental paradigms (e.g., Rajalakshmi \& Jeeves, 1965; Slotnick \& Brosvic, 1987). Such findings may also be related to effects associated with behavioral momentum, as described by Nevin and Grace (2000). Because wolves participated in both Experiments 1 and 2, a contingency reversal for the wolves in the blind condition of Experiment 2 was a necessary by-product of the methodological design: Wolves that had previously been reinforced for approaching the seer in Experiment 1 could now only obtain reinforcement by approaching the blind experimenter. While measures were taken to reduce the effects of this prior reinforcement history, including the presentation of only 10 bucket-condition trials in Experiment 1 and a break of at least 9 months between Experiments 1 and 2, it is still possible that even this small amount of direct experience resulted in wolves that were less likely to beg from the blind experimenter during Experiment 2. While this is a potential limitation, it is important to note that the naive dogs tested in Experiment 2 showed the same behavioral trend as the wolves without any prior experimental exposure to the task. Namely, dogs were more likely to learn to beg reliably from the seer as opposed to the person with the bucket over her head, even if begging from this blind experimenter was the only behavior that had ever been experimentally reinforced. It is also possible that this method of assignment might have put wolves at a disadvantage in the seer group as well, in comparison to their naive pet dog counterparts, possibly contributing to the slightly lower percentage of wolves learning to beg from the seer $(50 \%)$ in comparison to pet dogs $(80 \%)$. When possible, future studies should attempt to carry out such learning tests with naive wolves, to reduce the possibility of additional reversal effects; unfortunately, such a design was not possible in this case.

It is also a possibility that the failure of the dogs to beg preferentially from the blind experimenter was due to reversal-learning effects, but instead of a reversal with respect to prior experimental testing, this would have to be 
a reversal stemming from the natural contingencies established in the dog's home environment. Given this possibility, 20 trials might not have been sufficient to overcome the prior reinforcement history of the subjects in this study. Future studies are needed to determine whether this potential effect can be reversed, either by increasing the number of exposure trials or, possibly, through the implementation of a more explicit training procedure, such as the one utilized by Elgier, Jakovcevic, Mustaca, and Bentosela (2009) with dogs in a human point-reversal experiment.

Nevertheless, explanations that consider the relevant reinforcement histories of the subjects fit the overall pattern of data from Experiment 2 better than alternatives that might assume that subjects consistently avoided the person with the bucket over her head due to aversive qualities or fear. For canids in the blind condition, where only going to the blind experimenter was reinforced, the seer was still favored, but only slightly: On average, canids chose the seer $51 \%$ of the time (median 50\%), while choosing the blind experimenter $38 \%$ of the time (median $40 \%$ ). No-choice responses were recorded for roughly $11 \%$ of the trials (median $5 \%$ ). Only 1 subject in the blind condition, Jake, never chose the blind experimenter, and only 1 subject in the blind condition, Moon, never chose the seer. Therefore, with a greater number of trials, it seems plausible that canids could learn to avoid the seer and to preferentially beg from the blind experimenter in this context; however, additional research is needed to be sure of this.

These results demonstrate that, as in pointing tasks (Udell et al., 2008a), domestication is not required, nor is it sufficient, to explain the performance of domesticated dogs on perspective-taking tasks. Furthermore, while an associative account cannot directly prove the absence of underlying cognitive processes, nor can it directly negate a traditional theory-of-mind account, in this study additional cognitive processes were not required in order to predict or explain the performance of canids on this task. Instead, our findings favor the two-stage hypothesis, according to which "Dogs' ability to follow human actions stems from a willingness to accept humans as social companions, acquired early in ontogeny, combined with conditioning to follow the limbs and actions of humans to acquire reinforcement" (Udell et al., 2010b, p. 328). The outcome of this study is wholly consistent with previous findings that pet dogs are sensitive to human attentional states in a number of different contexts (Gácsi et al., 2004; Virányi, Topál, Gácsi, Miklósi, \& Csányi 2004), while showing more sensitivity to some attentional cues than to others (Cooper et al., 2003), and that whether dogs obey a command given by a human is not only dependent on attentional cues (Bräuer, Call, \& Tomasello 2004; Call,
Bräuer, Kaminski, \& Tomasello 2003; Schwab \& Huber, 2006) but the context in which the command is typically presented (Fukuzawa, Mills, \& Cooper 2005).

Such considerations are equally important when asking about the social skills and perspective-taking abilities of other animal species, including humans. It is not enough to ask whether a species demonstrates theory of mind or advanced social cognition; one has to specify the conditions under which this may occur. We have demonstrated here and elsewhere (see Udell et al., 2008a) that certain populations of domestic dogs include individuals that do not demonstrate spontaneous sensitivity to human social stimuli. It is also interesting to note that these underperforming populations often comprise dogs with a reduced level of exposure to humans. It might be especially valuable to assess the behavioral response of feral dogs to human stimuli, although this raises its own experimental and practical challenges. Nonetheless, the failure of some dogs to utilize human stimuli in predictable ways does not negate the fact that many pet domestic dogs excel in humanguided tasks. Likewise, the fact that some wolves have failed to use human cues in previous research (Hare et al., 2002) does not demonstrate lack of capacity; other socialized wolves have succeeded in following human points (Gácsi et al., 2009; Udell et al., 2008a; Virányi et al., 2008) and now in perspective-taking tasks. While demonstrating that a species or population has a capacity for a specified behavior is important, it is only a beginning. Understanding the conditions that foster the display of a behavior is just as valuable. Future studies should put more emphasis on understanding variables such as context and experience when assessing a canid's ability to utilize human actions and attentional cues.

\section{References}

Baum, W. M. (1998). Why not ask "Does the chimpanzee have a soul?". The Behavioral and Brain Sciences, 21, 116.

Bentosela, M., Barrera, G., Jakovcevic, A., Elgier, A. M., \& Mustaca, A. E. (2008). Effect of reinforcement, reinforcer omission and extinction on a communicative response in domestic dogs (Canis familiaris). Behavioural Processes, 78, 464-469. doi:10.1016/j. beproc.2008.03.004.

Bräuer, J., Call, J., \& Tomasello, M. (2004). Visual perspective taking in dogs (Canis familiaris) in the presence of barriers. Applied Animal Behaviour Science, 88, 299-317. doi:10.1016/j.applanim. 2004.03.004.

Call, J., \& Tomasello, M. (2008). Does the chimpanzee have a theory of mind? 30 years later. Trends in Cognitive Sciences, 12, 187192. doi:10.1016/j.tics.2008.02.010.

Call, J., Bräuer, J., Kaminski, J., \& Tomasello, M. (2003). Domestic dogs (Canis familiaris) are sensitive to the attentional state of humans. Journal of Comparative Psychology, 117, 257-263. doi:10.1037/0735-7036.117.3.257. 
Cooper, J. J., Ashton, C., Bishop, S., West, R., Mills, D. S., \& Young, R. J. (2003). Clever hounds: Social cognition in the domestic dog (Canis familiaris). Applied Animal Behaviour Science, 81, 229244. doi:10.1016/S0168-1591(02)00284-8.

Elgier, A. M., Jakovcevic, A., Mustaca, A. E., \& Bentosela, M. (2009). Learning and owner-stranger effects on interspecific communication in domestic dogs (Canis familiaris). Behavioural Processes, 8, 44-49. doi:10.1016/j.beproc.2008.12.023.

Fukuzawa, M., Mills, D. S., \& Cooper, J. J. (2005). More than just a word: Non-semantic command variables affect obedience in the domestic dog (Canis familiaris). Applied Animal Behaviour Science, 91, 129-141. doi:10.1016/j.applanim. 2004.08.025.

Gácsi, M., Miklósi, A., Varga, O., Topál, J., \& Csányi, V. (2004). Are readers of our face readers of our minds? Dogs (Canis familiaris) show situation-dependent recognition of human's attention. Animal Cognition, 7, 144-153. doi:10.1007/s10071003-0205-8.

Gácsi, M., Gyori, B., Virányi, Z., Kubinyi, E., Range, F., Belenyi, B., \& Miklósi, A. (2009). Explaining dog wolf differences in utilizing human pointing gestures: Selection for synergistic shifts in the development of some social skills. PLoS ONE, 4. doi:10.1371/journal.pone.0006584

Gómez, J. C. (1998). Assessing theory of mind with nonverbal procedures: Problems with training methods and an alternative "key" procedure. The Behavioral and Brain Sciences, 21, 119120.

Hare, B., \& Tomasello, M. (2005). Human-like social skills in dogs? Trends in Cognitive Sciences, 9, 439-444. doi:10.1016/j. tics.2005.07.003.

Hare, B., Brown, M., Williamson, C., \& Tomasello, M. (2002). The domestication of social cognition in dogs. Science, 298, 1634 1636. doi:10.1126/science. 1072702 .

Hare, B., Rosati, A., Kaminski, J., Brauer, J., Call, J., \& Tomasello, M. (2010). The domestication hypothesis for dogs' skills with human communication: A response to Udell et al. (2008) and Wynne et al. (2008). Animal Behaviour, 79, E1-E6. doi:10.1016/ j.anbehav.2009.06.031.

Heyes, C. M. (1998). Theory of mind in nonhuman primates. The Behavioral and Brain Sciences, 21, 101-114. doi:10.1017/ S0140525X98000703.

Kaminski, J., Call, J., \& Tomasello, M. (2004). Body orientation and face orientation: Two factors controlling apes' begging behavior from humans. Animal Cognition, 7, 216-223. doi:10.1007/ s10071-004-0214-2.

Klinghammer, E., \& Goodman, P. A. (1987). Socialization and management of wolves in captivity. In H. Frank (Ed.), Man and wolf: Advances, issues, and problems in captive wolf research (pp. 31-61). Dordrecht, The Netherlands: W. Junk.

Miklósi, Á., Polgárdi, R., Topál, J., \& Csányi, V. (2000). Intentional behaviour in dog-human communication: An experimental analysis of "showing" behaviour in the dog. Animal Cognition, 3, 159-166. doi:10.1007/s100710000072.

Miklósi, Á., Topál, J., \& Csányi, V. (2007). Big thoughts in small brains? Dogs as a model for understanding human social cognition. Neuroreport, 18, 467-471. doi:10.1097/ WNR.0b013e3280287aae.

Miklósi, Á., Kubinyi, E., Topál, J., Gácsi, M., Virányi, Z., \& Csányi, V. (2003). A simple reason for a big difference wolves do not look back at humans, but dogs do. Current Biology, 13, 763-766. doi:10.1016/S0960-9822(03)00263-X.

Miller, H. C., Rayburn-Reeves, R., \& Zentall, T. R. (2009). Imitation and emulation by dogs using a bidirectional control procedure. Behavioural Processes, 80, 109-114. doi:10.1016/j.beproc. 2008.09.011.
Nevin, J. A., \& Grace, R. C. (2000). Behavioral momentum and the Law of Effect. The Behavioral and Brain Sciences, 23, 7390.

Penn, D. C., \& Povinelli, D. J. (2007). On the lack of evidence that non-human animals possess anything remotely resembling a "theory of mind. Philosophical Transactions of the Royal Society B, 362, 731-744. doi:10.1098/rstb.2006.2023.

Petter, M., Musolino, E., Roberts, W. A., \& Cole, M. (2009). Can dogs (Canis familiaris) detect human deception? Behavioural Processes, 82, 109-118. doi:10.1016/j.beproc.2009.07.002.

Povinelli, D. J., \& Eddy, T. J. (1996). What young chimpanzees know about seeing. Monographs of the Society for Research in Child Development, 61(3), 1-191.

Povinelli, D. J., \& Vonk, J. (2003). Chimpanzee minds: Suspiciously human? Trends in Cognitive Sciences, 7, 157-160. doi:10.1016/ S1364-6613(03)00053-6.

Rajalakshmi, R., \& Jeeves, M. (1965). The relative difficulty of reversal learning (reversal index) as a basis of behavioural comparisons. Animal Behaviour, 13, 203-211. doi:10.1016/ 0003-3472(65)90035-7.

Range, F., Virányi, Z., \& Huber, L. (2007). Selective imitation in domestic dogs. Current Biology, 17, 868-872. doi:10.1016/j. cub.2007.04.026.

Riedel, J., Schumann, K., Kaminski, J., Call, J., \& Tomasello, M. (2008). The early ontogeny of human-dog communication. Animal Behaviour, 75, 1003-1014. doi:10.1016/j.anbehav. 2007.08.010

Schwab, C., \& Huber, L. (2006). Obey or not obey? Dogs (Canis familiaris) behave differently in response to attentional states of their owners. Journal of Comparative Psychology, 120, 169-175. doi:10.1037/0735-7036.120.3.169.

Scott, J. P., \& Fuller, J. L. (1965). Genetics and the social behavior of the $\operatorname{dog}$ (1st ed.). Chicago: University of Chicago Press.

Slotnick, B. M., \& Brosvic, G. M. (1987). Failure of rats to acquire a taste reversal learning set. Chemical Senses, 12, 333-339. doi:10.1093/chemse/12.2.333.

Topál, J., Byrne, R. W., Miklósi, Á., \& Csányi, V. (2006a). Reproducing human actions and action sequences: "Do as I do!" in a dog. Animal Cognition, 9, 355-367. doi:10.1007/ s10071-006-0051-6.

Topál, J., Erdõhegyi, Á., Mányik, R., \& Miklósi, Á. (2006b). Mindreading in a dog: An adaptation of a primate "mental attribution" study. International Journal of Psychology and Psychological Therapy, 6, 365-379.

Udell, M. A. R., \& Wynne, C. D. L. (2008). A review of domestic dogs' (Canis familiaris) human-like behaviors: Or why behavior analysts should stop worrying and love their dogs. Journal of the Experimental Analysis of Behavior, 89, 247-261. doi:10.1901/ jeab.2008.89-247.

Udell, M. A. R., Dorey, N. R., \& Wynne, C. D. L. (2008a). Wolves outperform dogs in following human social cues. Animal Behaviour, 76, 1767-1773. doi:10.1016/j.anbehav. 2008.07.028.

Udell, M. A. R., Giglio, R. F., \& Wynne, C. D. L. (2008b). Domestic dogs (Canis familiaris) use human gestures but not nonhuman tokens to find hidden food. Journal of Comparative Psychology, 122, 84-93. doi:10.1037/0735-7036.122.1.84.

Udell, M. A. R., Dorey, N. R., \& Wynne, C. D. L. (2010a). The performance of stray dogs (Canis familiaris) living in a shelter on human-guided object-choice tasks. Animal Behaviour, 79, $717-$ 725. doi:10.1016/j.anbehav.2009.12.027.

Udell, M. A. R., Dorey, N. R., \& Wynne, C. D. L. (2010b). What did domestication do to dogs? A new account of dogs' sensitivity to human actions. Biological Reviews, 85, 327-345. doi:10.1111/ j.1469-185X.2009.00104.x. 
Virányi, Z., Topál, J., Gácsi, M., Miklósi, Á., \& Csányi, V. (2004). Dogs respond appropriately to cues of humans' attentional focus. Behavioural Processes, 66, 161-172. doi:10.1016/j.beproc.2004.01.012.

Virányi, Z., Gácsi, M., Kubinyi, E., Topál, J., Belényi, B., Ujfalussy, D., et al. (2008). Comprehension of human pointing gestures in young human-reared wolves (Canis lupus) and dogs (Canis familiaris). Animal Cognition, 11, 373-387. doi:10.1007/s10071007-0127-y.
Author note We thank Dana Drenzek, Patricia Goodman, Holly Jaycox, Erich Klinghammer, Ken McCort, Gale Motter, Monty Sloan, and Tom O'Dowd at Wolf Park, Battle Ground, Indiana, for their expert help and participation. We also thank Janet Hobson for letting us use the facilities at Dogwood Park, the staff and director of the Alachua County Animal Services, and all of the individual dog owners who made this research possible. We also thank three anonymous reviewers for their comments on an earlier draft of the manuscript. 